\title{
A influência do tratamento medicamentoso na qualidade de vida de diabéticos
}

\section{The influence of drug treatment on the QoL of people with diabetes \\ La influencia del tratamiento farmacológico para la calidad de vida de personas con diabetes}

\author{
Claudete Moreschi iD \\ Universidade Regional Integrada do Alto Uruguai e das Missões - Santiago (RS) - Brasil
}

Daiana Foggiato de Siqueira (iD

Universidade Federal de Santa Maria Santa Maria - Santa Maria (RS) - Brasil

Luís Felipe Pissaia iD

Universidade do Vale do Taquari - Lajeado (RS) - Brasil

Dirce Stein Backes iD

Universidade Franciscana - Santa Maria (RS) - Brasil

Carmen Neri Fernández Pombo iD

Universidade de Vigo - Vigo - Pontevedra - Espanha

Claudete Rempel (iD

Universidade do Vale do Taquari - Lajeado (RS) - Brasil

\section{RESUMO}

Objetivo: Analisar a adesão de pessoas diabéticas ao tratamento medicamentoso e sua relação com a qualidade de vida. Métodos: Estudo transversal desenvolvido com 350 pessoas com diabetes no município de Lajeado, Rio Grande do Sul. A coleta de dados ocorreu por meio dos questionários Medida de Adesão ao Tratamento (MAT) e Whoqol-bref, aplicados no domicílio dos participantes durante o ano de 2015. Resultados: A maioria das pessoas com diabetes investigadas toma o antidiabético oral metformina ( $n=305 ; 87,1 \%)$, seguido de glibenclamida $(n=151 ; 43,1 \%)$, e $20,9 \%(n=73)$ fazem uso de insulina. A maioria toma sinvastatina $(n=259 ; 74 \%)$, ácido acetilsalicílico (AAS) $(n=203 ; 58 \%)$, enalapril $(n=192 ; 55 \%)$ e hidroclorotiazida ( $n=180$; $52 \%$ ). Essa população adere positivamente ao tratamento medicamentoso relativo ao controle da doença e a média geral de qualidade de vida é boa $(67,6 \pm 18,1)$. A média de qualidade de vida das pessoas que aderem ao tratamento medicamentoso é maior $(68,6 \pm 15,9)$ do que as que não aderem $(60,9 \pm 14,2)(t=3,3162 ; p=0,0012)$. Conclusão: Constatou-se nessa amostra de diabéticos que os que têm melhor adesão ao tratamento medicamentoso relativo ao controle da doença também apresentam uma melhor qualidade de vida quando comparados com o grupo que tem menor adesão aos medicamentos.

Descritores: Diabetes Mellitus; Atenção Básica à Saúde; Promoção da saúde.

\section{ABSTRACT}

Objective: To analyze adherence to drug treatment in people with diabetes and its relationship to quality of life. Methods: This cross-sectional study was carried out with 350 people with diabetes in the municipality of Lajeado, Rio Grande do Sul, Brazil. Data were collected through the Measurement of Adherence to Treatment (MAT) and the WHOQoL-bref questionnaires, which were administered at the participants' homes during the year 2015. Results: Most of the people with diabetes analyzed take the oral antidiabetic drug metformin ( $n=305 ; 87.1 \%)$, followed by glibenclamide ( $n=151 ; 43.1 \%)$, and $20.9 \%$ ( $n=73)$ use insulin. The majority takes simvastatin ( $n=259 ; 74 \%)$, acetylsalicylic acid (ASA) ( $n=203 ; 58 \%)$, enalapril ( $n=192 ; 55 \%)$, and hydrochlorothiazide ( $n=180 ; 52 \%)$. This population group positively adheres to the drug treatment regime for the control of the disease the mean overall quality of life is good (67.6 \pm 18.1$)$. The mean quality of life score among people who adhere to drug the treatment is higher (68.6 \pm 15.9$)$ compared to those who do not $(60.9 \pm 14.2)(t=3.3162 ; p=0.0012)$. Conclusion: In this sample of people with diabetes, those who exhibited good adherence to the drug treatment regime for the control of the disease also presented better quality of life compared to those who exhibited poor adherence to the drug treatment.

Descriptors: Diabetes Mellitus; Primary Health Care; Health Promotion. 


\section{RESUMEN}

Objetivo: Analizar la adhesión de personas con diabetes para el tratamiento farmacológico y su relación con la calidad de vida. Métodos: Estudio transversal desarrollado con 350 personas con diabetes del municipio de Lajeado, Río Grande de Sur. La recogida de datos se dio a través de los cuestionarios Medida de Adhesión al Tratamiento (MAT) y el Whoqol-bref que han sido aplicados en el domicilio de los participantes durante el año 2015. Resultados: La mayoría de las personas con diabetes investigadas toma el fármaco oral para la diabetes metformina ( $n=305 ; 87,1 \%)$, seguido del glibenclamida $(n=151 ; 43,1 \%)$ y el 20,9\% ( $n=73)$ usa la insulina. La mayoría toma la sinvastatina ( $n=259 ; 74 \%)$, el ácido acetilsalicílico (AAS) ( $n=203 ; 58 \%)$, el enalapril $(n=192 ; 55 \%)$ y la hidroclorotiazida $(n=180 ; 52 \%)$. Esa población tiene adhesión al tratamiento farmacológico para el control de la enfermedad y la media general de la calidad de vida es buena $(67,6 \pm 18,1)$. La media de la calidad de vida de las personas que tiene adhesión al tratamiento farmacológico es mayor $(68,6 \pm 15,9)$ de que las que no adhieren al tratamiento $(60,9 \pm 14,2)(t=3,3162 ; p=0,0012)$. Conclusión: En esa muestra de diabéticos se constató que los que tienen mejor adhesión al tratamiento farmacológico para el control de la enfermedad también tienen mejor calidad de vida comparándolos con el grupo de menor adhesión a los fármacos.

Descriptores: Diabetes Mellitus; Atención Primaria de Salud; Promoción de la Salud.

\section{INTRODUÇÃO}

O diabetes mellitus (DM) é considerado uma doença ou síndrome metabólica que possui como característica a incapacidade do organismo de produzir insulina ou de sintetizá-la, ambas as etiologias podem ocorrer num mesmo

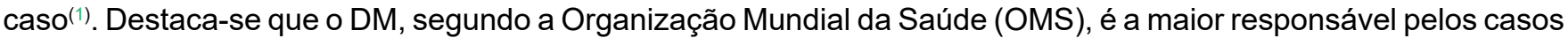
de óbito e debilidades no mundo(2). Entre os principais agravos decorrentes dessa doença estão os episódios de hipoglicemia e hiperglicemia, sendo este último responsável por danos teciduais em longo prazo em órgãos, como o coração e os rins ${ }^{(3)}$.

Devido à alta demanda de pessoas com DM na atenção básica e seus gastos decorrentes com internações e medicações, o Ministério da Saúde (MS) verificou a necessidade de reorganização da assistência oferecida a essa população específica ${ }^{(4)}$. Visando à efetividade das ações em saúde, instituiu-se o Plano Nacional de Reorganização da Atenção à Hipertensão Arterial e ao Diabetes Mellitus, instrumentalizada pelas Estratégias Saúde da Família (ESF)(5).

A assistência prestada a esse público exige um longo período de adaptação ao tratamento, desenvolvendo o autocuidado, auxílio da família, acompanhamento e realização de vigilância em saúde pela Atenção Primária à Saúde (APS $)^{(6)}$. O tratamento do DM realiza-se por meio de intervenções que visam à autoaceitação da doença, com medidas medicamentosas e não medicamentosas ${ }^{(7)}$.

As medidas medicamentosas, incluindo antidiabéticos orais e a aplicação de insulina, bem como a prescrição dessas medicações, são realizadas por profissionais capacitados e não são necessárias a todos os pacientes com $\mathrm{DM}^{(7)}$. Já as medidas não medicamentosas são incentivadas a todos os portadores. Como exemplo, temos adesão a hábitos alimentares saudáveis, a realização de atividades físicas, a monitorização dos níveis de glicemia e restrição ao uso de álcool e fumo ${ }^{(8)}$.

A adesão ao tratamento influencia na qualidade de vida da pessoa com DM por diminuir os riscos de complicações associadas à patologia, considerando a compreensão da doença pela pessoa como a principal forma de conscientização social. Os profissionais da saúde possuem os instrumentos de educação em saúde como aliados na compreensão mútua de sua comunidade sobre boas práticas em saúde, buscando, além da promoção da saúde, a efetividade da assistência às pessoas que possuem $\mathrm{DM}^{(9)}$.

É importante considerar que a QV está relacionada com a subjetividade e a multidimensionalidade, bem como com a presença de dimensões positivas e negativas de cada pessoa. Nessa lógica, segundo o conceito da OMS, entende-se por QV "a percepção do indivíduo de sua posição na vida no contexto de sua cultura e no sistema de valores em que vive e em relação as suas expectativas, seus padrões e suas preocupações". Considera-se este um conceito amplo, uma vez que incorpora, de forma complexa, a saúde física, o estado psicológico, o nível de independência, as relações sociais, as crenças pessoais e a relação com aspectos significativos do meio ambiente ${ }^{(10)}$.

Sustentada numa perspectiva transcultural, a OMS construiu um instrumento de avaliação de QV denominado World Health Organization Quality of Life (WHOQOL). Esse instrumento contempla a subjetividade da avaliação, a qual está relacionada ao contexto cultural, social e ambiental. Avalia-se a percepção do respondente/pessoa e não a natureza objetiva do meio ambiente, do estado funcional ou do estado psicológico, ou mesmo como o profissional de saúde ou um familiar avalia esses aspectos ${ }^{(11)}$. 
Desse modo, pautado na cientificidade, o estudo em questão tem como objetivo analisar a adesão de pessoas diabéticas ao tratamento medicamentoso e sua relação com a qualidade de vida, buscando-se subsídios para o fortalecimento das práticas em saúde, a fim de que se possa contribuir com os programas para a promoção da saúde e melhoria na assistência às pessoas com DM.

\section{MÉTODOS}

Trata-se de um estudo transversal envolvendo 350 pessoas com DM em acompanhamento nas 14 Estratégias de Saúde da Família (ESFs) de um município do interior do estado do Rio Grande do Sul. Levou-se em consideração o número de casos de DM cadastrados em 2013 no Sistema de Informação da Atenção Básica (SIAB) para compor a amostra do estudo. Nesse período, no município do estudo, foram cadastradas 1.125 pessoas com DM, com 18 anos ou mais. Sendo assim, a composição de uma amostra com $95 \%$ de confiança e $4,4 \%$ de erro resultou em 350 participantes da avaliação.

O município em questão possui 71.445 habitantes, sendo considerado altamente urbanizado, com 99,9\% da população na área urbana, ou seja, 71.180 habitantes ${ }^{(12)}$. Foram selecionados, de forma aleatória, em cada uma das 14 ESF, 25 indivíduos, com idade superior a 18 anos, para participação no estudo. A coleta de dados ocorreu durante $o$ ano de 2015 nos domicílios dos participantes.

Coletaram-se as informações por meio de questionário, elaborado pelos autores, abordando o perfil epidemiológico dos indivíduos portadores de DM, constituído por variáveis sociodemográficas e contemplando os medicamentos utilizados pela população estudada. As variáveis sociodemográficas analisadas no questionário do perfil epidemiológico incluem: idade, sexo, cor, ocupação (trabalho), nível de escolaridade, estado civil, religião, renda mensal familiar e as medicações utilizadas.

Utilizou-se, ainda, o questionário Medida de Adesão ao Tratamento (MAT), que avalia a adesão ao tratamento medicamentoso, e o questionário Whoqol-bref para avaliar a QV dos pesquisados. O questionário MAT é composto por sete itens, cujas respostas, dadas na escala Likert, variam de 1 a 6 pontos, em que 1 corresponde a sempre e o 6 a nunca. A soma dos valores conseguidos é dividida por sete, número referente à quantidade de perguntas do MAT. Aos valores finais 5 e 6 é atribuído o conceito "1" (aderente na escala original) e aos demais valores é atribuído o conceito zero (não aderente na escala original), apresentando a escala dicotômica sim (1)/ não(0) (aderente/não aderente ao uso dos medicamentos) ${ }^{(13)}$.

O Whoqol-bref é uma escala para medir QV em adultos numa perspectiva transcultural, considerando como características essenciais o caráter subjetivo da QV e sua natureza multidimensional. É formado por questões autoaplicáveis e aborda a percepção dos sujeitos em seu contexto, considerando sua cultura e seus valores sobre sua QV. Através dele, a pessoa entrevistada realiza uma reflexão acerca das duas últimas semanas de sua vida e avalia como se sente quanto à sua QV, tendo em vista seus valores, aspirações, prazeres e preocupações, assinalando somente uma das cinco alternativas apresentadas para cada uma das questões ${ }^{(11)}$.

O WHOQOL-bref envolve 26 questões, nomeadas facetas, nas quais são investigados quatro domínios: físico, psicológico, ambiental e das relações sociais. A determinação dos escores dos domínios do WHOQOL-brefé realizada em etapas. Primeiramente, soma-se todos os itens incluídos dentro de um domínio e, após, divide-se pela média de questões respondidas. Posteriormente, multiplica-se o resultado por quatro. Na segunda etapa, diminui-se de cada domínio o valor de quatro e multiplica-se por 6,25 (ou 100/16). Assim, o resultado final de cada domínio vai variar de 0 a 100. Para melhor compreensão da QV das pessoas com DM, utiliza-se a escala Likert, dividida em cinco classes de igual tamanho: muito ruim (0-20); ruim (21-40); nem ruim nem boa (41-60); boa (61-80); muito boa (80-100) ${ }^{(11)}$.

A aplicação dos questionários ocorreu no momento da visita domiciliar. Para aqueles com dificuldades, realizou-se a leitura do instrumento de pesquisa e auxiliou-se no preenchimento das respostas apontadas.

As respostas aos questionários (socioeconômico, MAT e Whoqol-bref) foram tabuladas em planilhas do Microsoft Excel. Realizou-se a análise dos dados socioeconômicos por meio de estatística descritiva, utilizando-se o software Statistical Package for the Social Sciencies - SPSS, versão 21, sendo os dados apresentados na forma de frequência relativa (\%) e absoluta. Para os questionários MAT e o Whoqol, utilizou-se a sintaxe prevista para cada um, sendo os dados gerados na própria planilha do Excel. Os resultados são demonstrados por meio de gráficos e tabelas, além de média (desviopadrão). Para verificar a diferença estatística na média de escore de QV das pessoas que aderem e não aderem ao tratamento, realizou-se o teste "t" para amostras independentes no software SPSS.

A pesquisa recebeu autorização da Secretaria Municipal de Saúde do município de estudo e do Comitê de Ética em Pesquisa (COEP) do Centro Universitário Univates, sob o Parecer $n .^{\circ} 997.286$. Foram respeitados os critérios éticos previstos pela Resolução n. ${ }^{\circ} 466$, de 12 de dezembro de 2012, do Conselho Nacional de Saúde ${ }^{(14)}$, que define as diretrizes e normas reguladoras de pesquisas envolvendo seres humanos. 


\section{RESULTADOS}

Verificou-se que, das 350 pessoas com DM, a maioria $(n=238 ; 68,0 \%)$ é do sexo feminino e sua média de idade é de $64,4( \pm 11,2)$ anos. A faixa etária que apresentou maior prevalência foi de 60 a 69 anos, com percentual de $36,6 \%$ ( $n=128)$. Quanto ao estado civil, a maioria (64,9\%) é casada. A maioria das pessoas possui ensino fundamental incompleto $(68,6 \% ; n=240), 11,7 \%(n=41)$ dessa população não sabe ler nem escrever, e apenas $0,3 \%$ $(n=1)$ possuem ensino superior completo.

Quanto à ocupação, 75,2\% ( $n=241)$ são aposentados ou pensionistas, 9,7\% ( $n=34)$ do lar, 4\% autônomos $(n=14)$. A cor branca, entre as pessoas com DM, prevaleceu em $85,7 \%(n=300)$. Quanto à renda mensal familiar, $21,7 \%(n=76)$ recebem até um salário, $46 \%(n=161)$ de um a dois salários, $28,6 \%(n=100)$ de dois a quatro salários e $3,7 \%(n=13)$ de quatro a 10 salários.

A identificação dos medicamentos utilizados será apresentada na Figura 1.

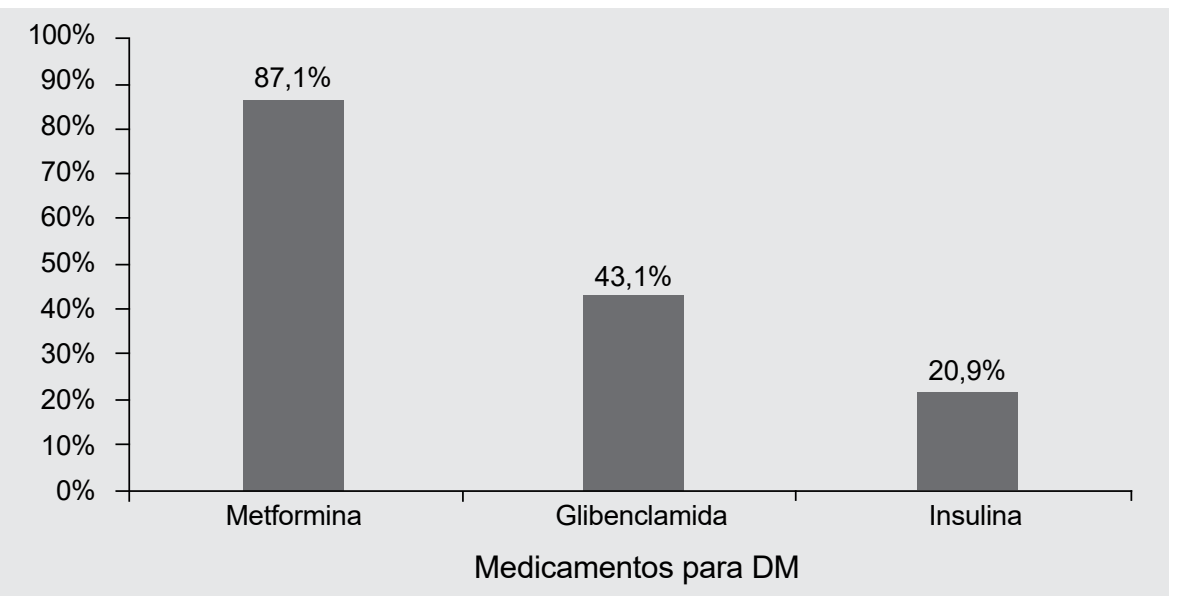

Figura 1 - Medicamentos utilizados pelas pessoas atendidas nas Estratégias Saúde da Família (ESFs) para o tratamento do diabetes mellitus (DM). Lajeado, Rio Grande do Sul, Brasil, 2015.

Verificou-se, também, que os participantes tomam medicamentos para outras doenças associadas, estando entre os medicamentos mais utilizados sinvastatina, seguido de ácido acetilsalicílico (AAS), enalapril e hidroclorotiazida (Figura 2). Essa população também faz uso de algumas medicações com menor prevalência, entre elas: anlodipino, diazepan, digoxina, metropolol, atenolol, sertralina, atensina, cabomazepina, izorbida e amiodarona.

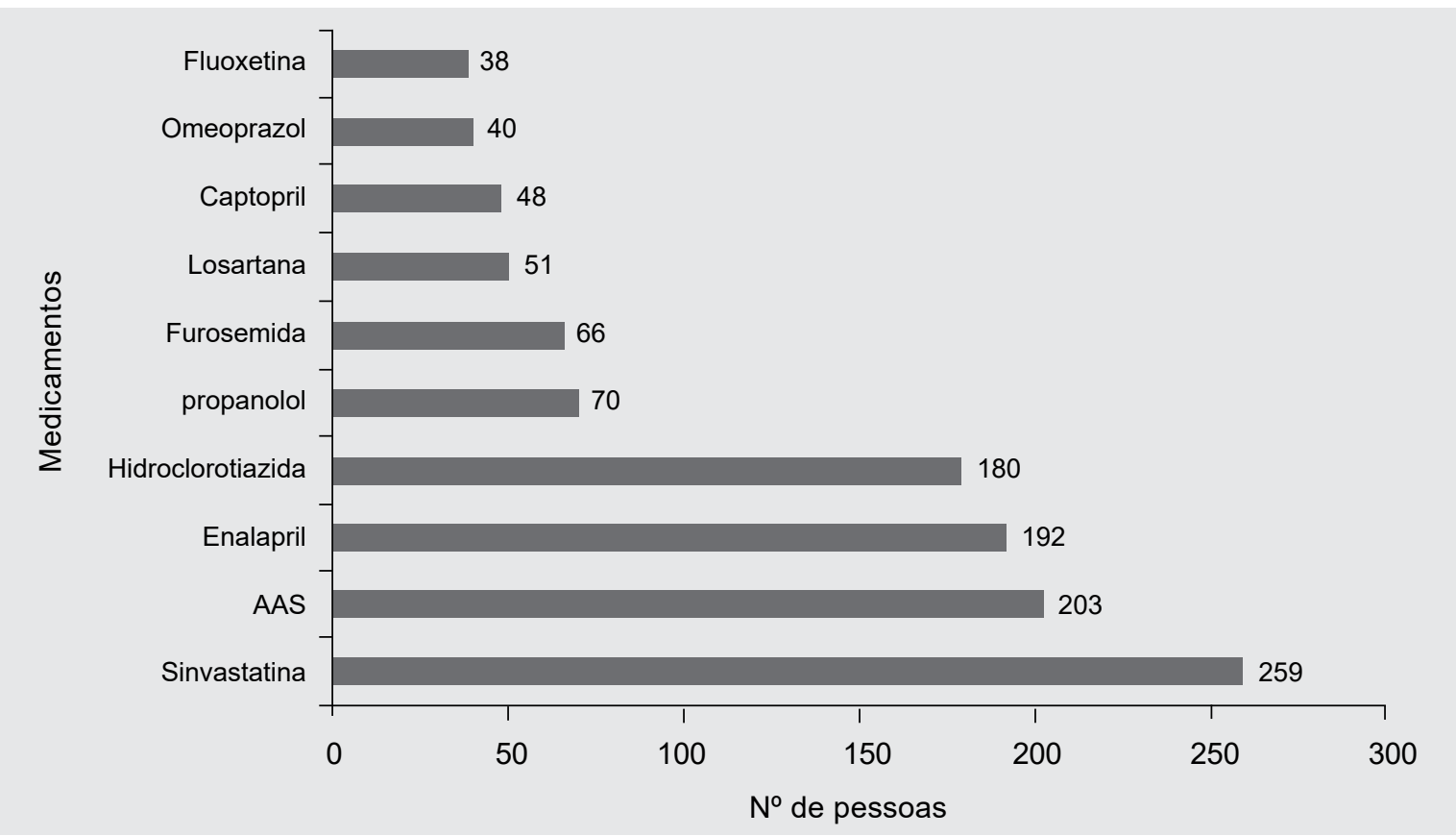

Figura 2 - Medicamentos que mais fazem uso para outras doenças associadas as pessoas com diabetes mellitus atendidas nas Estratégias Saúde da Família (ESFs). Lajeado, Rio Grande do Sul, Brasil, 2015. 
Ao se analisar a adesão ao tratamento medicamentoso da amostra por meio do Questionário MAT, verificou-se que a média de adesão da amostra foi de $5,56( \pm 0,49)$, numa escala pontuada de 1 a 6 . Aderiram ao tratamento $86,9 \%$ da amostra e 13,1\% não têm adesão satisfatória ao tratamento medicamentoso.

Ao avaliar a QV por meio da aplicação do questionário Whoqol-bref, verificouse que a média geral de QV foi de $67,6( \pm 18,1)$ pontos, ou seja, em média, os participantes apresentavam uma boa QV. Mesmo ao dividir a amostra nos grupos que aderem e que não aderem ao tratamento medicamentoso, ambos os grupos apresentaram boa QV, com 68,6 $( \pm 15,9)$ e 60,9 $( \pm 14,2)$, respectivamente, demonstrando diferença estatística na $Q V(t=3,3162 ; p=0,0012)$.

\section{DISCUSSÃO}

A adesão ao tratamento medicamentoso de pessoas com diabetes mellitus constitui-se num valioso indicador para avaliar a efetividade da atenção em diabetes, uma vez que apresenta relevância clínica para os profissionais de saúde, possibilitando direcionar a tomada de decisões clínicas em relação ao tratamento do diabetes na equipe da ESF(15).

Quanto ao tratamento medicamentoso, verificou-se que a maioria dos participantes do presente estudo toma metformina (87,1\%). O segundo medicamento mais utilizado foi a glibenclamida $(43,1 \%)$, e $20,8 \%$ fazem uso de insulina. Esses achados corroboram outros estudos do Sul do Brasil, em que as medicações mais utilizadas são as mesmas do estudo em questão, sendo sempre a insulina a menos utilizada nos estudos em questão(16,17).

O Plano de Reorganização da Atenção à Hipertensão Arterial e ao Diabetes Mellitus promoveu a reestruturação e a ampliação do atendimento resolutivo e de qualidade para os portadores dessas condições. Desse modo, o Ministério da Saúde adotou como padrão de tratamento do DM medicamentos essenciais, preconizados pela Organização Mundial de Saúde (OMS), sendo os hipoglicemiantes orais, como a glibenclamida, em comprimidos de $5 \mathrm{mg}$, e a metformina, em comprimidos de $850 \mathrm{mg}$, além da insulina NPH 100Ul, disponibilizados na rede de saúde brasileira de forma sistemática e gratuita ${ }^{(18)}$.

Pode-se evidenciar, ainda, que as pessoas com DM deste estudo tomam medicamentos para outras doenças associadas. Os medicamentos que a maioria toma são sinvastatina, seguido de AAS, enalapril e hidroclorotiazida, os quais são usados para tratar dislipidemia, doenças cardiovasculares e hipertensão. Esses fatores, comumente associados com o DM, podem ser controlados $^{(2)}$. Em estudo realizado num município do interior da Bahia sobre o uso de medicamentos e estilo de vida no gerenciamento do DM em idosos, evidenciou-se que $70 \%$ dos indivíduos pesquisados eram hipertensos e usavam medicação para tal patologia ${ }^{(19)}$.

Em relação à adesão ao tratamento medicamentoso das pessoas com DM atendidas nas ESF (questionário MAT) do atual estudo, constatou-se que a maioria das pessoas investigadas no presente estudo adere o tratamento $(86,9 \%)$. Estudo desenvolvido em Minas Gerais, com pacientes com DM tipo 2 atendidos nas ESF, constatou adesão ao tratamento medicamentoso superior a $60 \%$ em todas as ESF investigadas ${ }^{(15)}$. Outros estudos nacionais realizados em amostras populacionais com DM também encontraram boa adesão ao tratamento medicamentoso, respectivamente, $78,3 \%$ e $97,6 \%{ }^{(1,16)}$.

No presente estudo, a média obtida para a adesão ao tratamento medicamentoso representou um valor de 5,56 em uma escala pontuada de 1 a 6 , na qual escores mais altos indicam maior adesão. Portanto, observa-se que a média está próxima do valor máximo possível, podendo-se dizer que a amostra estudada possui uma boa adesão medicamentosa. A adesão positiva ao tratamento medicamentoso pode estar relacionada às percepções e crenças das pessoas com DM sobre o seu tratamento. O tratamento é composto por três modalidades: medicação, dieta e exercício físico; porém, geralmente, as pessoas consideram que a medicação é o tipo de tratamento de maior importância no controle do $\mathrm{DM}^{(20)}$.

Portanto, as porcentagens elevadas estão vinculadas à aceitabilidade do tratamento medicamentoso e à crença no efeito do medicamento para o controle da doença ${ }^{(20)}$. Essa evidência também pode estar relacionada com a política de distribuição de medicamentos gratuitos pela rede de saúde, que garante sua acessibilidade ${ }^{(3,21)}$.

Outros estudos relatam que um dos maiores desafios encontrados pelos profissionais de saúde é a baixa adesão das pessoas com diabetes ao tratamento, no qual se insere o tratamento medicamentoso(19,22). Dados que não corroboram os achados do presente estudo. Cabe destacar que o questionário MAT contém perguntas que se referem ao uso adequado da medicação, horário, quantidade, motivo para não tomarem etc., no período dos últimos sete dias, apresentando as possibilidades de resposta para cada questão(13,23).

Ao comparar a média de QV das pessoas com DM e a adesão ao tratamento medicamentoso, houve diferença significativa entre a média da QV das pessoas que aderem o tratamento e a média das que não aderem na atual pesquisa. Isso permite inferir que as pessoas que aderem ao tratamento medicamentoso possuem QV melhor do 
que as que não aderem. A baixa adesão ao tratamento medicamentoso é um dos motivos primários que levam à redução da estabilidade clínica das pessoas com DM, contribuindo com o surgimento de complicações de saúde e psicossociais, o que diminui a QV dos indivíduos. Desse modo, considerando o evidenciado neste estudo, de que as pessoas que aderem o tratamento medicamentoso apresentam uma melhor QV do que as não aderentes, faz-se necessário que as equipes multiprofissionais das ESF desenvolvam ações educativas ${ }^{(23,24)}$. Essa equipe precisa abranger o uso correto dos medicamentos relativos ao tratamento do DM, sobretudo orientar as pessoas que apresentam algum tipo de dificuldade em aderir ao tratamento medicamentoso ${ }^{(25,26)}$.

É relevante que os profissionais de saúde promovam educação em saúde voltada para a compreensão das pessoas com DM da importância da QV, bem como da adesão a todo o tratamento. Os profissionais precisam levar em consideração que a baixa adesão está vinculada à resistência às mudanças nos hábitos de vida e à dificuldade dos indivíduos entenderem e se adaptarem ao tratamento medicamentoso ${ }^{(27)}$. Considera-se importante destacar que a não adesão ao tratamento do DM não pode ser observada apenas como uma incompetência da pessoa em seguir seu tratamento de maneira apropriada, pois também deve ser vista como uma possível falha do sistema de saúde em oferecer os cuidados adequados aos usuários conforme suas singularidades ${ }^{(20,27)}$.

Recomenda-se que o plano terapêutico da pessoa com DM aconteça com a participação ativa do sujeito. Precisa-se estabelecer uma relação de confiança e credibilidade entre profissional e usuário, oferecendo um cuidado individualizado e multiprofissional. Assim, o indivíduo vai se sentir mais motivado ao tratamento e ser persistente nele. Nessa perspectiva, as práticas de educação em saúde ocupam espaços importantes, uma vez que podem contribuir na sensibilização das pessoas com DM para a adesão farmacológica e a adequação de seus hábitos e condutas ${ }^{(4,7,28)}$.

Entende-se que o presente estudo apresentou como limitação o fato de ter sido desenvolvido em apenas um município do estado do Rio Grande do Sul o que pode limitar a generalização dos resultados obtidos. Além do mais, consideram-se as limitações em decorrência do delineamento transversal, no qual a coleta de dados da exposição e do desfecho ocorre em um único momento.

Contudo, o impacto deste estudo se traduz na construção de evidências sobre o tratamento do DM, que tem por objetivo manter o controle glicêmico, a promoção da saúde e melhorar a qualidade de vida dos indivíduos. Para isso, é indispensável que as pessoas acometidas por essa doença crônica realizem o tratamento medicamentoso de forma adequada e, assim, consequentemente, poderão diminuir as adversidades durante a terapia e impedir o agravamento da doença e possíveis complicações decorrentes ${ }^{(28-30)}$.

\section{CONCLUSÃO}

Ao analisar a adesão ao tratamento medicamentoso, relacionando-a com a qualidade de vida de pessoas com diabetes, constatou-se que a população deste estudo adere positivamente ao tratamento medicamentoso relativo ao controle da doença. A média geral de qualidade de vida é boa, sendo significativamente maior nas que aderem ao tratamento medicamentoso.

\section{AGRADECIMENTOS}

À Fundação de Amparo à Pesquisa do Estado do Rio Grande do Sul (FAPERGS).

\section{CONTRIBUIÇÕES}

Claudete Morechi e Claudete Rempel contribuíram com a elaboração e delineamento do estudo; a aquisição, análise e interpretação de dados; e a redação e/ou revisão do manuscrito. Dirce Stein Backes, Carmen Neri Fernández Pombo, Luís Felipe Pissaia e Daiana Foggiato de Siqueira contribuíram com a aquisição, análise e interpretação de dados; e a redação e/ou revisão do manuscrito. Todos os autores aprovaram a versão final do manuscrito e são responsáveis por todos seus aspectos, incluindo a garantia de sua precisão e integridade.

\section{REFERÊNCIAS}

1. Sociedade Brasileira de Diabetes. Diretrizes da Sociedade Brasileira de Diabetes 2017-2018. São Paulo: Editora Clannad; 2017.

2. World Health Organization. Diabetes [Internet]. Geneva: WHO; 2013 [acesso em 2017 Jul 19]. Disponível: www.who. int/topics/diabetes_mellitus/en 
3. Greghi EFM, Pascon DM. Knowledge of diabetes mellitus type 2 patients inserted into the adult health care program. Rev Fac Ciênc Méd Sorocaba [Internet]. 2016 [acesso em 2017 Jun 26];18(4):204-9. Disponível: https:// www.researchgate.net/publication/313231779_Conhecimento_dos_pacientes_portadores_de_ diabetes_mellitus_tipo_2_inseridos_no_Programa_de_Saude_ao_Adulto

4. Costa KS, Tavares NUL, Mengue SS, Pereira MA, Malta DC, Silva JB Jr. Obtaining drugs for hypertension and diabetes through the Brazilian People's Pharmacy Program: results of the National Health Survey, 2013. Epidemiol Serv Saúde [Internet]. 2016 [acesso em 2017 Ago 10];25(1):33-44. Disponível: http://www.scielo.br/ pdf/ress/ v25n1/2237-9622-ress-25-01-00033.pdf

5. Ministério da Saúde (BR). Plano de reorganização da atenção à hipertensão arterial e ao diabetes mellitus [Internet]. Brasília: Ministério da Saúde; 2002. [acesso em 2016 Ago 10]. Disponível: http://bvsms.saude.gov. br/bvs/publicacoes /miolo2002.pdf

6. Dutra DD, Duarte MCS, Albuquerque KF, Lima AS, Santos JS, Souto HC. Cardiovascular disease and associated factors in adults and elderly registered in a basic health unit. Rev Pesqui (Univ Fed Estado Rio J) [Internet]. 2016 [acesso em 2016 Ago 08];8(2):4501-9. Disponível: http://search.ebscohost.com/login. aspx?dire $c t=t r u e \& d b=$ foh \&AN=114759934(=pt-br\&site=eds-live

7. Alves MS, Araújo MDCF, Nascimento MP, Ribeiro FC, Rebouças PT, Santos TA, et al. Grupo Terapêutico com idosos sobre o autocuidado nas doenças crônicas. J Health Sci [Internet]. 2016 [acesso em 2017 Jun 18];18(1):52-5. Disponível: http://pgsskroton.com.br/seer/index.php/JHealthSci/article/viewFile/3515/3107

8. Machado JC, Cotta RMM, Moreira TR, Silva LSD. Analysis of three health education strategiesfor patients with arterial hypertension. Ciênc Saúde Colet [Internet]. 2016 [acesso em 2017 Jun 18];21(2):611-20. Disponivel: http:// www.scielo.br/scielo.php?script=sci_arttextmd=S1413-81232016000200611

9. Lima TAM, Fazan ER, Pereira LLV, Godoy MF. Acompanhamento farmacoterapêutico em idosos. Arq Ciênc Saúde [Internet]. 2016 [acesso em 2017 Jun 18];23(1):52-7. Disponível: http://www.cienciasdasaude.famerp. br/index. php/racs/article/view/229/176

10. The WHOQOL Group. The World Health Organization quality of life assessment (WHOQOL): position paper from the World Health Organization. Soc Sci Med. 1995;41:1403-9.

11. Fleck MPA. O instrumento de avaliação de qualidade de vida da OMS (WHOQOL-100): características e perspectivas. Ciênc Saúde Colet [Internet]. 2000 [acesso em 2017 Jun 18];5(1):33-8. Disponível: http://www. scielo.br/ pdf/csc/v5n1/7077.pdf

12. Instituto Brasileiro de Geografia e Estatística. XII Censo Demográfico [Internet]. 2010 [acesso em 2017 Mar 12]. Disponível: http:www.ibge.gov.br

13. Delgado $A B$, Lima ML. Contributo para validação concorrente de uma medida de adesão aos tratamentos. Psicol Saúde Doenças [Internet]. 2001 [acesso em 2016 Jun 18];2(2):81-100. Disponível: http://www.scielo. mec.pt/scielo.php?script=sci_arttext\&pid=S1645-00862001000200006

14. Ministério da Saúde (BR). Diretrizes e normas regulamentadoras sobre pesquisa envolvendo seres humanos: resolução 466. Brasília: Ministério da Saúde; 2012.

15. Faria HTG, Santos MA, Arrelias CCA, Rodrigues FFL, Gonela JT, Teixeira CRS, et al. Adesão ao tratamento em diabetes mellitus em unidades da Estratégia Saúde da Família. Rev Esc Enferm USP [Internet]. 2014 [acesso em 2017 Jun 18];48(2):257-63. Disponível: http://www.scielo.br/pdf/reeusp/v48n2/pt_0080-6234reeusp-48-02-257.pdf

16. Carvalho CV, Rocha LP, Carvalho DP, Silva BT, Oliveira SM, Silveira RB. Adesão de pessoas com diabetes mellitus tipo II ao tratamento medicamentoso. Rev Enferm UFPE. 2017 [acesso em 2017 Jun 18];11(9):34029.

17. Silva AB, Engroff $P$, Sgnaolin V, Ely LS, Gomes I. Prevalência de diabetes mellitus e adesão medicamentosa em idosos da Estratégia Saúde da Família de Porto Alegre/RS. Cad Saúde Colet. 2016;24(3):308-16.

18. Ministério da Saúde; Organização Pan-Americana da Saúde. Avaliação do Plano de Reorganização da Atenção à Hipertensão Arterial e ao Diabetes Mellitus no Brasil. Brasília: Ministério da Saúde; 2004.

19. Santos-Souza A, Cardoso-Santos IS, Campos-Meira E, Oliveira BBC, Souza-Júnior EV. Uso de 
medicamentos e estilo de vida no gerenciamento do diabetes em idosos. Rev Salud Pública. 2019;21(3)1-7.

20. Boell JEW, Silva DMGVD, Guanilo MEE, Hegadoren K, Meirelles BHS, Suplici SR. Resiliência e autocuidado em pessoas com diabetes mellitus. Texto \& Contexto Enferm. 2020;29.

21. Farias EBO, Espinheira MJCL, Santana NRS, Rodrigues RLA. Adesão terapêutica em pacientes diabéticos em uso medicamentoso. Rev Psicol. 2019;13(43)407-15.

22. Borba AKOT, Marques APO, Ramos VP, Leal MCC, Arruda IKG, Ramos RSPS. Fatores associados à adesão terapêutica em idosos diabéticos assistidos na atenção primária de saúde. Ciênc Saúde Colet. 2018;23(3):953-61.

23. Salin AB, Bandeira MSN, Freitas PRNDO, Serpa I. Diabetes Mellitus tipo 2: perfil populacional e fatores associados à adesão terapêutica em Unidades Básicas de Saúde em Porto Velho-RO. Rev Eletrônica Acervo Saúde. 2019;(33).

24. Reis P, Marcon SS, Nass EMA, Arruda GO, Back IR, Lino IGT, et al. Desempenho de pessoas com diabetes mellitus na insulinoterapia. Cogitare Enferm. 2020;25.

25. Silva AD, Matos N Jr, Damasceno DD, Guimarães NS, Gomes JMG. Estado nutricional, fatores de risco e comorbidades em adultos portadores de diabetes mellitus tipo 2. HU Rev. 2020;46:1-9.

26. Marcelo CAS, Coutinho MAP, Lara CR, Paraizo CMS, Fava SMCL. Aplicativos móveis sobre diabetes mellitus-Revisão Narrativa. J Health Inform. 2020;12(2).

27. Nogueira BCM, Souza CAD, Manzano RM, Rosa CSDC, Barrile SR, Ximenes MA, et al. Aspectos emocionais e autocuidado de pacientes com Diabetes Mellitus Tipo 2 em Terapia Renal Substitutiva. Cad Bras Ter Ocup. 2019;27(1):127-34.

28. Campos LS, Silva CB, Wanderley TLR, Candeia VMM, Calzerra NTM. A prática da atenção farmacêutica no acompanhamento farmacoterapêutico de idosos diabéticos e hipertensos: relato de caso. Braz J Health Rev. 2020;3(2):2287-96.

29. Santos WP, Silva MM, Freitas FBD, Souza FT. Interfaces da (não) adesão ao tratamento do diabetes mellitus tipo II. Rev Ciênc Saúde Nova Esperança. 2019;17(2)56-63.

30. Maia L, Lopes RR, Tomazetti D, Alves MM, Mariño PAP, Menezes APS. Polifarmácia: avaliação em grupo de hipertensos e diabéticos. Salão Int Ensino Pesqui Extensão. 2019;11(2).

\section{Endereço para correspondência:}

Claudete Moreschi

Universidade Regional Integrada do Alto Uruguai e das Missões - Campus Santiago

Av. Batista Bonoto Sobrinho, 733

CEP: 97700-000 - Santiago (RS) - Brasil

E-mail: clau_moreschi@!yahoo.com.br

Como citar: Moreschi C, Siqueira DF, Pissaia LF, Backes DS, Pombo CNF, Rempel C. A influência do tratamento medicamentoso na qualidade de vida de diabéticos. Rev Bras Promoç Saúde. 2020;33:10125. 